\title{
Safety and Immunogenicity of Four-Segmented Rift Valley Fever Virus in the Common Marmoset
}

Paul Wichgers Schreur ( $\nabla$ paul.wichgersschreur@wur.nl)

Wageningen Bioveterinary Research https://orcid.org/0000-0001-9790-2438

\section{Petra Mooij}

Biomedical Primate Research Centre

\section{Gerrit Koopman}

Biomedical Primate Research Institute

\section{Babs Verstrepen}

BPRC https://orcid.org/0000-0002-4666-0798

\section{Zahra Fagrouch}

Biomedical Primate Research Centre

\section{Daniella Mortier}

Biomedical Primate Research Institute

\section{Nikki van Driel}

Biomedical Primate Research Centre

\section{Jet Kant}

Wageningen Bioveterinary Research

\section{Sandra van de Water}

Wageningen Bioveterinary Research

\section{Willy Bogers}

Biomedical Primate Research Institute https://orcid.org/0000-0002-4563-8513

\section{Carine Punt}

BunyaVax

\section{Lucien van Keulen}

Wageningen Bioveterinary Research, Houtribweg 39, 8221 RA Lelystad

\section{Ernst Verschoor}

Biomedical Primate Research Centre https://orcid.org/0000-0003-0912-2074

\section{Jeroen Kortekaas}

Wageningen Bioveterinary Research

Article 
Keywords: Rift Valley fever virus, vaccine, four-segmented, nonhuman primate, common marmoset, safety,

Posted Date: December 8th, 2021

DOI: https://doi.org/10.21203/rs.3.rs-1075403/v1

License: (1) This work is licensed under a Creative Commons Attribution 4.0 International License. Read Full License

Version of Record: A version of this preprint was published at npj Vaccines on May 18th, 2022. See the published version at https://doi.org/10.1038/s41541-022-00476-y. 
Safety and immunogenicity of four-segmented Rift Valley fever virus in the common marmoset

Short title: Safety and efficacy of RVFV-4s in marmosets

Paul J. Wichgers Schreur ${ }^{1,2^{\star}}$, Petra Mooij ${ }^{3}$, Gerrit Koopman ${ }^{3}$, Babs E. Verstrepen ${ }^{3}$, Zahra Fagrouch ${ }^{3}$, Daniella Mortier $^{3}$, Nikki van Driel ${ }^{3,}$ Jet Kant ${ }^{1}$, Sandra van de Water ${ }^{1}$, Willy M. Bogers ${ }^{3}$, Carine Punt ${ }^{2}$, Lucien van Keulen ${ }^{1}$, Ernst J. Verschoor ${ }^{3}$, Jeroen Kortekaas ${ }^{1,4}$

${ }^{1}$ Department of Virology and Molecular Biology, Wageningen Bioveterinary Research, Wageningen University \& Research, Lelystad, The Netherlands

${ }^{2}$ BunyaVax B.V., Lelystad, The Netherlands

${ }^{3}$ Department of Virology, Biomedical Primate Research Centre, Rijswijk, The Netherlands ${ }^{4}$ Laboratory of Virology, Wageningen University \& Research, Wageningen, The Netherlands.

*Corresponding author: paul.wichgersschreur@wur.nl

\section{Abstract}

Rift Valley fever virus (RVFV) is an emerging mosquito-borne bunyavirus that is highly pathogenic to wild- and domesticated ruminants, camelids and humans. While animals are exclusively infected via mosquito bites, humans can also be infected via contact with tissues or blood released during the slaughtering of RVFV-infected animals. No human vaccine is available and currently commercialized veterinary vaccines do not optimally combine efficacy with safety. We previously reported the development of two novel live-attenuated RVF vaccines, created by splitting the $\mathrm{M}$ genome segment and deleting the major virulence determinant NSs. The vaccine candidates, referred to as the veterinary vaccine vRVFV-4s and the human vaccine hRVFV-4s, were shown to induce protective immunity in multiple species after a single vaccination. Anticipating on accidental exposure of humans to the veterinary vaccine, and to evaluate the safety of the hRVFV-4s candidate vaccine for humans, the safety of each vaccine was evaluated in the most susceptible nonhuman primate model, the common marmoset (Callithrix jacchus). Marmosets were inoculated with high doses of each vaccine and were monitored for clinical signs as well as for vaccine virus dissemination, shedding and spreading to the environment. To accurately assess the attenuation of both vaccine viruses, separate groups of marmosets were inoculated with the parent wild-type RVFV strains. Both wild-type 
strains induced high viremia and disseminated to primary target organs, associated with mild- to severe morbidity, while both vaccines were well tolerated with absence of dissemination and shedding, while inducing potent neutralizing antibody responses. The results of the studies support the unprecedented safety profile of both vaccines for animals and humans.

Keywords: Rift Valley fever virus; vaccine; four-segmented; nonhuman primate; common marmoset; safety;

\section{Introduction}

Rift Valley fever virus (RVFV) belongs to the order Bunyavirales (genus Phlebovirus, family Phenuiviridae) and is the causative agent of Rift Valley fever (RVF). The mosquito-borne virus affects wild- and domesticated ruminants, such as sheep, goats, cattle, buffalo, and camelids, manifesting with liver necrosis and haemorrhagic manifestations, with fatal outcomes especially in young animals. Outbreaks are characterized by abortion storms in sheep herds. In endemic areas, epizootics are generally associated with explosive mosquito populations following heavy rains. A wide range of mosquito species have been associated with transmission of the virus, particularly those within the genera Aedes and Culex ${ }^{1,2}$. The virus is currently widespread in Africa and is expected to expand its habitat further, stimulated by climate change and globalization ${ }^{3}$.

In addition to infecting ruminants, RVFV is also infectious to humans. Humans can be exposed to the virus via contact with contaminated tissues or blood released during the slaughtering of RVFV-infected animals, or via mosquito bites. Although humans generally develop a self-limiting febrile illness, a significant fraction develops neurological disorders or haemorrhagic fever ${ }^{4}$, which is often fatal. Furthermore, increasing evidence suggests that RVFV infection during human pregnancies may result in complications ${ }^{5-7}$. Presently there are no vaccines or therapeutics available to prevent or treat human infections.

The RVFV genome is divided over three RNA segments of negative polarity that are named after their size; small (S), medium (M) and large (L). The $L$ segment encodes the viral RNA-dependent RNA polymerase. The S segment encodes the nucleocapsid that protects the viral RNA from degradation and is involved in transcription and replication, and a non-structural protein named NSs. NSs interferes with host innate immune signalling pathways and is considered the major virulence determinant of the virus ${ }^{8}$. The $\mathrm{M}$ segment encodes a polyprotein precursor that is co-translationally cleaved by host proteases into the structural glycoproteins $\mathrm{Gn}$ and $\mathrm{Gc}$. Gn is involved in attachment to target cells and $\mathrm{Gc}$ is required for fusion of the viral and endosomal membranes. The $\mathrm{M}$ segment additionally encodes a small 14-kDa protein, named NSm, which counteract apoptosis ${ }^{9}$, and a large 78- 
kDa glycoprotein, named LGp, that comprises the NSm and Gn coding regions, shown to be important for dissemination of the virus in mosquitoes ${ }^{10-12}$.

We previously reported the development of a novel live-attenuated RVF vaccine that was constructed by splitting the M genome segment into two M-type segments, one encoding $\mathrm{Gn}$ and the accessory proteins NSm and LGp, and one encoding Gc, resulting in a four-segmented RVFV (RVFV-4s) ${ }^{13}$. To optimize the safety profile, the NSs gene was deleted from the $S$ segment. The technology to create four-segmented RVF viruses was subsequently used to create tailor-made vaccines for both animal and human application. The veterinary vaccine, here referred to as "v"RVFV-4s, is based on strain 35/74 and optimally replicates in ruminant cells, whereas the vaccine to be applied in humans, here referred to as "h"RVFV-4s is based on the naturally attenuated Clone 13 isolate $^{14,15}$.

Both vRVFV-4s and hRVFV-4s were shown to be safe and efficacious in various rodent and ruminant animal models ${ }^{13,16-20}$, however, safety-data obtained with a more appropriate model for humans was lacking. Demonstration of safety in a nonhuman primate (NHP) model is not only relevant for the candidate human vaccine, but also for the candidate veterinary vaccine, as veterinarians applying this vaccine could be exposed accidentally to the vaccine virus. NHPs, considering their close phylogenetic relationship to humans, are one of the most predictive models with regard to human vaccine safety and efficacy ${ }^{21}$. Historically, rhesus macaques were used for the evaluation of candidate RVF vaccines and therapeutics, despite their relatively low susceptibility to the wild-type virus $^{22,23}$. More recently, common marmosets (Callithrix jacchus) were shown to provide a more convenient model, as these animals develop higher morbidity, more consistent viremia and marked aberrations in hematological and biochemistry values following wild-type RVFV infection compared to macaques ${ }^{24,25}$.

In this study, we evaluated safety and immunogenicity of a high dose of the vRVFV-4s vaccine candidate and of 3 doses of the hRVFV-4s vaccine candidate in the common marmoset. We specifically assessed body temperatures, virus dissemination and shedding, and induction of neutralizing antibody responses compared to their parent wild-type RVFV strains. 


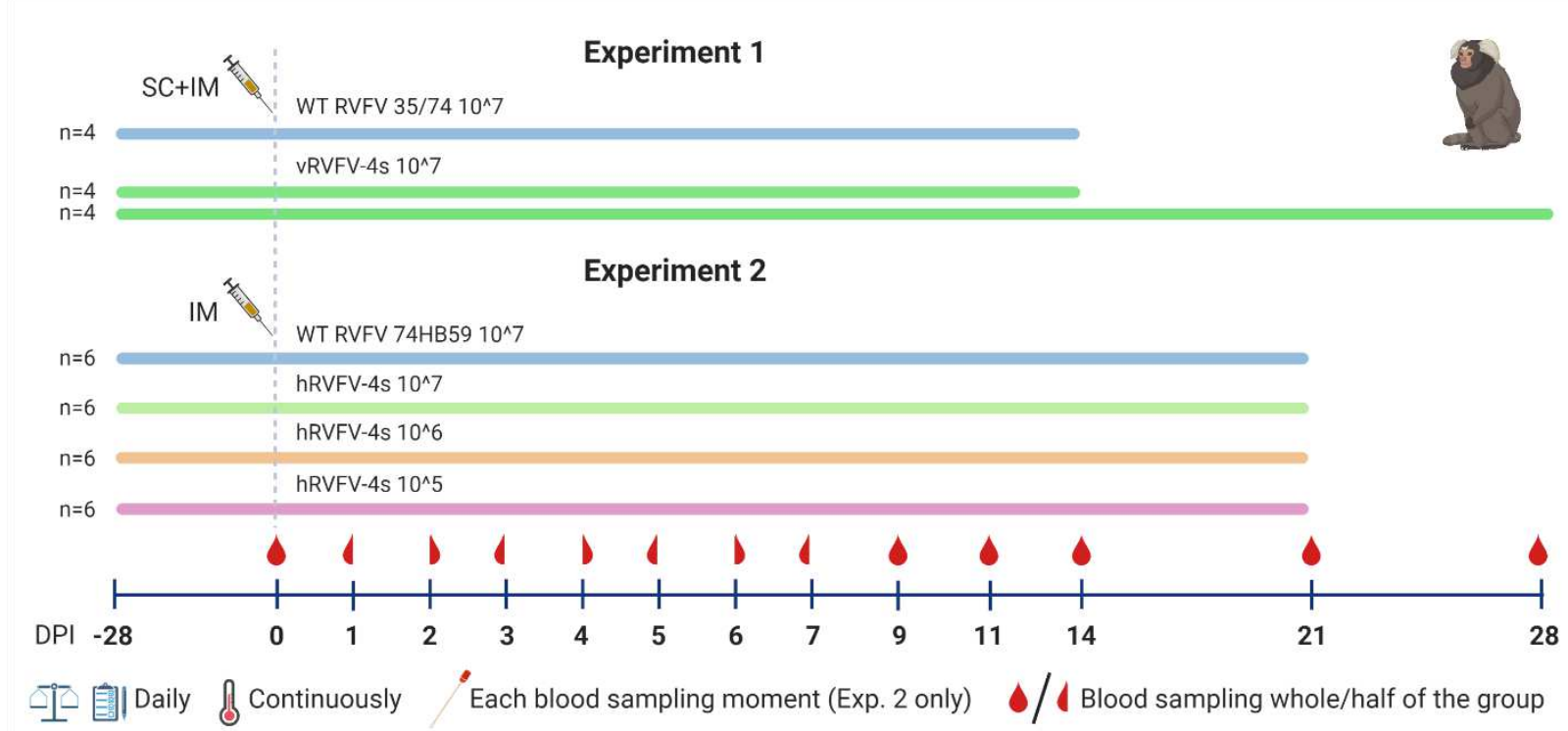

\section{Results}

\section{Experimental design}

To assess the safety and immunogenicity of both vRVFV-4s and hRVFV-4s in the marmoset model, we conducted two independent experiments (Fig. 1). In experiment 1, eight marmosets were inoculated with a dose of $10^{\wedge} 7 \mathrm{TCID}_{50}$ of vRVFV-4s via a combined intramuscular (IM) and subcutaneous (SC) route. On day 14 and day 28 , four inoculated animals were euthanized to collect organ samples. Another 4 marmosets were inoculated with a $10^{\wedge} 7$ $\mathrm{TCID}_{50}$ dose of parent strain 35/74, and were necropsied on day 14 . In experiment 2 , three groups of six animals were inoculated with either $10^{\wedge} 5,10^{\wedge} 6$ or $10^{\wedge} 7 \mathrm{TCID}_{50}$ of hRVFV-4s via IM route only as the IM route is the anticipated route for human vaccination. In this experiment, a group of identical size was inoculated with $10^{\wedge} 7$ $\mathrm{TCID}_{50}$ of virulent parent strain 74HB59. At three weeks post inoculation, all animals of experiment 2 were euthanized and the organs were collected. Animals in both experiments were frequently monitored for body weight and clinical signs, besides continuous monitoring of body temperatures. Furthermore, blood samples were regularly collected for haematological, biochemical, virological and immunological analyses. To assess potential virus shedding, oral and rectal swabs were taken in experiment 2.
95

Figure 1. Schematic representation of the experimental design. Marmosets of experiment 1 were inoculated with vRVFV-4s and in experiment 2 with hRVFV-4s with the indicated dose and route. In both experiments, control animals were inoculated with the wild-type parent strains; for vRVFV-4s this is strain 35/74 and for hRVFV-4s this is 74HB59. Blood samples were taken regularly as indicated, and body temperatures were monitored continuously by an abdominal implanted AniPillV2. Oral and rectal swabs were taken only during experiment 2 . 
None of the animals inoculated with vRVFV-4s (experiment 1) or hRVFV-4s (experiment 2) presented abnormal behaviour or manifested with clinical signs or weight loss during the course of the experiment (Fig. 2a, b). One out of four wild-type RVFV 35/74 inoculated animals (experiment 1, animal M12030) showed tremors starting on day 4 post inoculation. These clinical signs worsened on day 15 and the animal was euthanized consequently. This animal additionally presented with substantial weight loss following inoculation. Of note, this animal presented with some (unaccountable) weight loss already prior inoculation. The other three animals of this group did not show clinical signs and were euthanized at the end of the experiment. In experiment 2, approximately 11 days post inoculation (DPI), four out of six control animals inoculated with wild-type strain 74HB59 (M16010, M16017, M16040, and M17011) started to show clinical signs, such as a unkempt coats and hunched postures. The general health of animal M16010 and M17011 gradually improved over time, but at 13 and $14 \mathrm{DPI}$ the physical condition of marmosets M16040 and M16017 deteriorated to a lethargic state, reaching a pre-defined humane endpoint. Of note, animal M17058 presented with shallow breathing, unkempt coat and a hunched posture for 2 days starting at day 14 post inoculation. This animal recovered but maintained shallow breathing until the end of the study (day 21). Disease severity was more pronounced in marmosets inoculated with the 74HB59 strain compared to strain 35/74. No behavioural changes, clinical signs or weight loss were observed in any of the vRVFV-4s or hRVFV-4s animals.

a

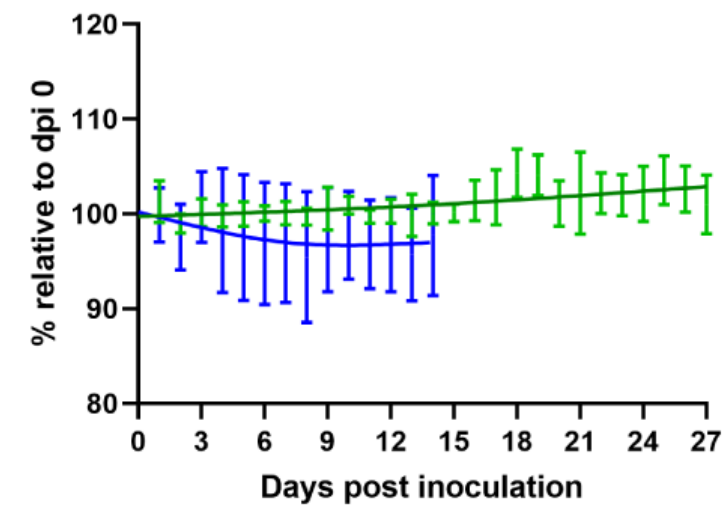

- WT RVFV-35/74 (10^7)

- vRVFV-4s $(10 \wedge 7)$ b

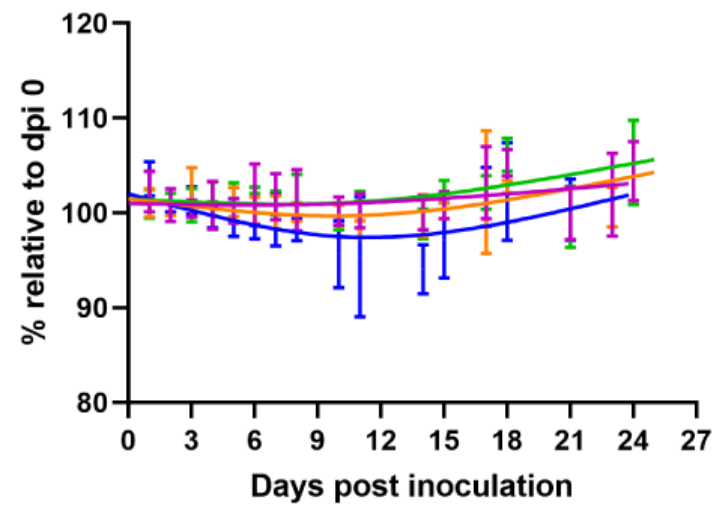

- WT RVFV-74HB59(10^7) - hRVFV-4s $(10 \wedge 6)$ hRVFV-4s $(10 \wedge 7) \quad$ hRVFV-4s $(10 \wedge 5)$

Figure 2. RVFV-4s inoculated animals demonstrate no significant weight loss. Body weights relatively to day post inoculation (DPI) 0 measured in experiment 1 (a) and experiment 2 (b). Standard deviations are presented at each time point and spline trendlines were added (Graphpad Prism 9.0). No statistically significant differences were observed between groups. 
123 Transient, dose-dependent body temperature increases in RVFV-4s inoculated marmosets

124 All animals were implanted with an Anipill, a telemetric device to continuously monitor the abdominal body 125 temperatures. Before inoculation, all animals showed a normal circadian pattern, with a body temperature of approximately $39^{\circ} \mathrm{C}$ during the day versus $36^{\circ} \mathrm{C}$ during the night. Following inoculation with either vRVFV-4s or hRVFV-4s, a consistent transient elevation of the night-time body temperatures was observed in all animals inoculated with a $10^{\wedge} 7 \mathrm{TCID}_{50}$ dose, starting at DPI 1, and persisting for approximately 24 hrs (Fig. 3). At lower doses $\left(10^{\wedge} 6\right.$ or $10^{\wedge} 5 \mathrm{TCID}_{50}$, Experiment 2), the transient elevation was less pronounced. Marmosets inoculated with the wild-type virus did not show this early-phase temperature increase but instead had a more pronounced temperature increase at 2-4 DPI that persisted on average for 1.5-2 days (Fig. 3). 
Experiment 1

a

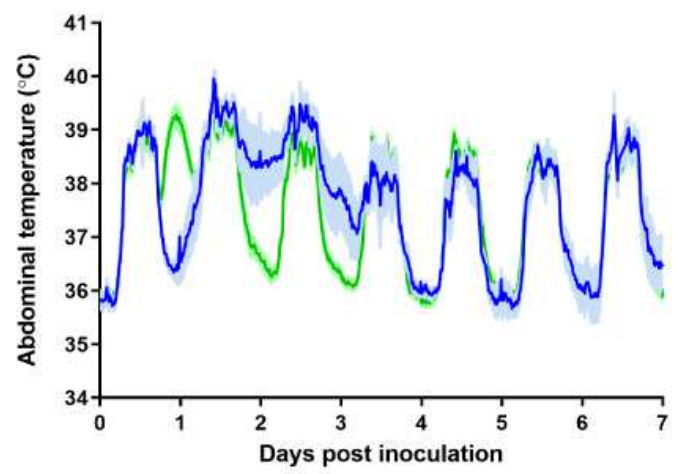

Experiment 1

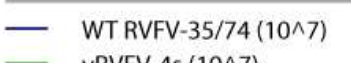

C

Temp. analysis 0.8-1.4 DPI Temp. analysis 1.5-3.3 DPI

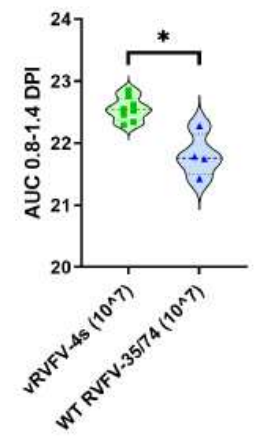

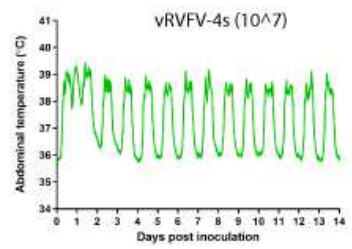

Experiment 2

- WT RVFV-74HB59(10^7)

- hRVFV-4s $(10 \wedge 7)$

- hRVFV-4s $\left(10^{\wedge} 6\right)$

- hRVFV-4s $\left(10^{\wedge} 5\right)$

Experiment 2
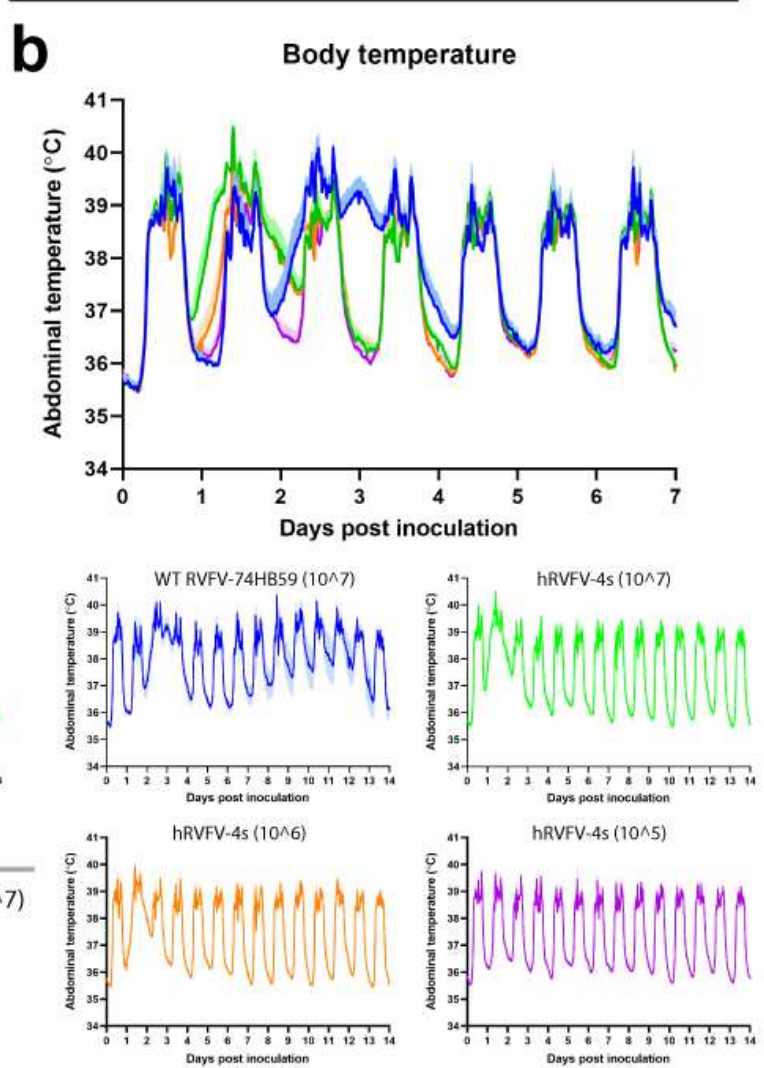

d
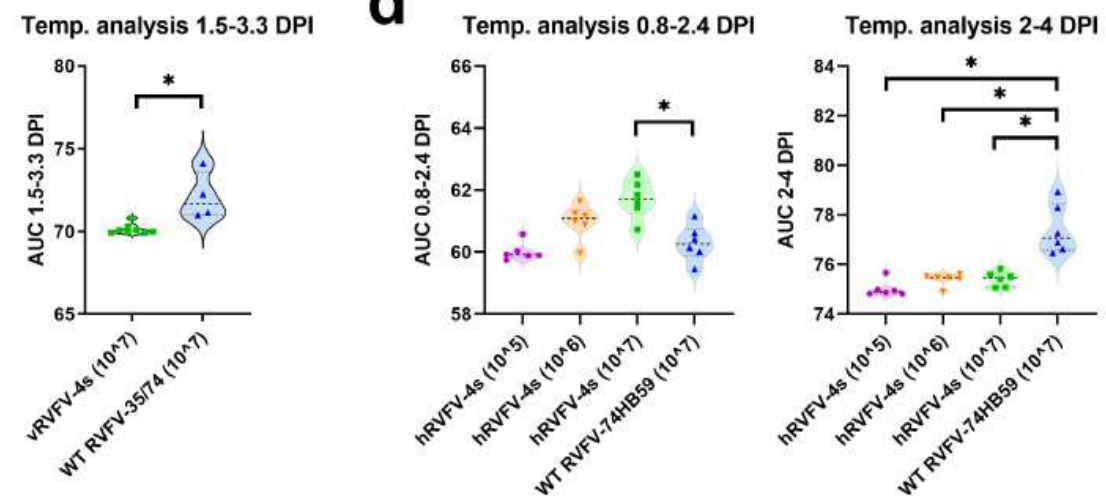

Figure 3. Dose dependent early-phase temperature increase following inoculation of marmosets with RVFV-4s. Body temperatures in experiment 1 (a) and experiment 2 (b) were recorded every 15 min using an Anipill telemetric device in the abdominal cavity. Averages and SEM are presented for each group. Area under the curve (AUC) measurements were performed at the indicated days post inoculation (DPI) to assess statistical significant differences between groups of experiment 1 (c) and, groups of experiment 2 (d). Data were analysed by an unpaired T-test (GraphPad Prism 9.0) and statistical significance ( $p$ value $\leq 0.05)$ is indicated with an asterisk $(*)$. 
141 Following inoculation with wild-type strain 35/74 in experiment 1, both animals sampled on day 1 post inoculation

142 (M15045 and M15046) showed a minor increase in white blood cell (WBC) and neutrophil counts (Figure 4). No 143 changes were observed at other time points and no changes were observed in lymphocyte and monocyte counts.

144 In the vRVFV-4s inoculated animals all cell numbers remained within the normal range as observed with historic 145 control animals at all time points assessed. In experiment 2, the overall haematology pattern slightly differed 146 compared to experiment 1. The WBC and neutrophil counts at day 1 post inoculation ( 3 out of 3 animals assessed) 147 were elevated compared to day 0 following hRVFV-4s inoculation. The animals inoculated with $10^{\wedge} 7$ TCID 50 had 148 higher WBC and neutrophil counts compared to the groups inoculated with lower doses, suggesting a dose 149 response. No increases in WBC and neutrophil counts were observed in animals inoculated with the corresponding 150 wild-type strain. Furthermore, no differences were observed in lymphocyte or monocyte counts following inoculation of either wild-type strain 74HB59 or hRVFV-4s. parameters in both experiments. Following inoculation with the wild-type strains (experiment 1 and 2) no major changes were observed, except for animal M12030 which presented with increased liver enzymes and renal function parameters in plasma (Fig. 4). No changes in blood chemistry of the VRVFV-4s or hRVFV-4s inoculated animals were observed. 


\section{Experiment 1}

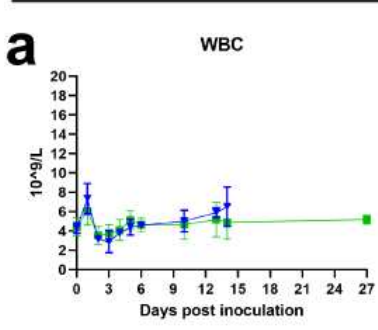

Lymphocytes

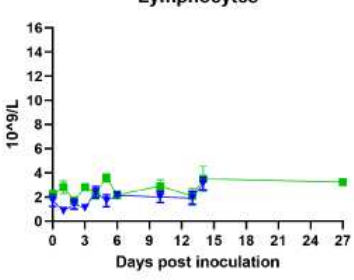

C

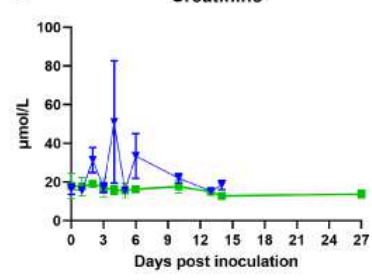

Alkaline Phosphatase

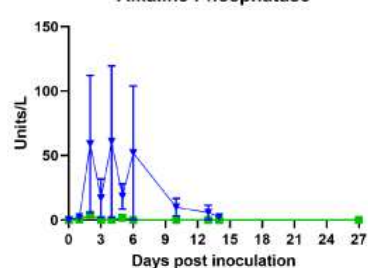
Days post inoculation

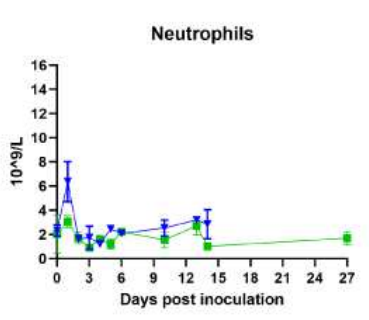

Monocytes

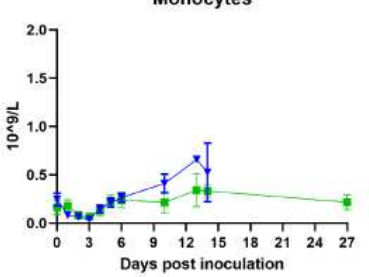

Alat

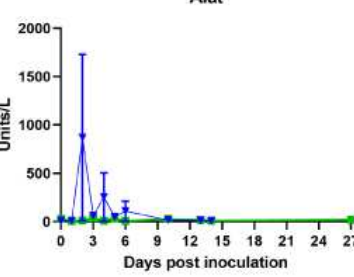

Urea

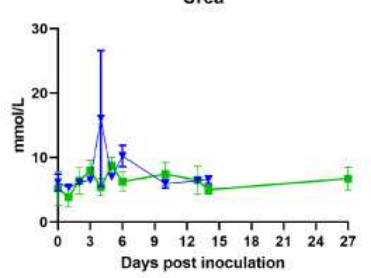

- WT RVFV-35/74 (10^7)

VRVFV-4s $\left(10^{\wedge} 7\right)$
Experiment 2
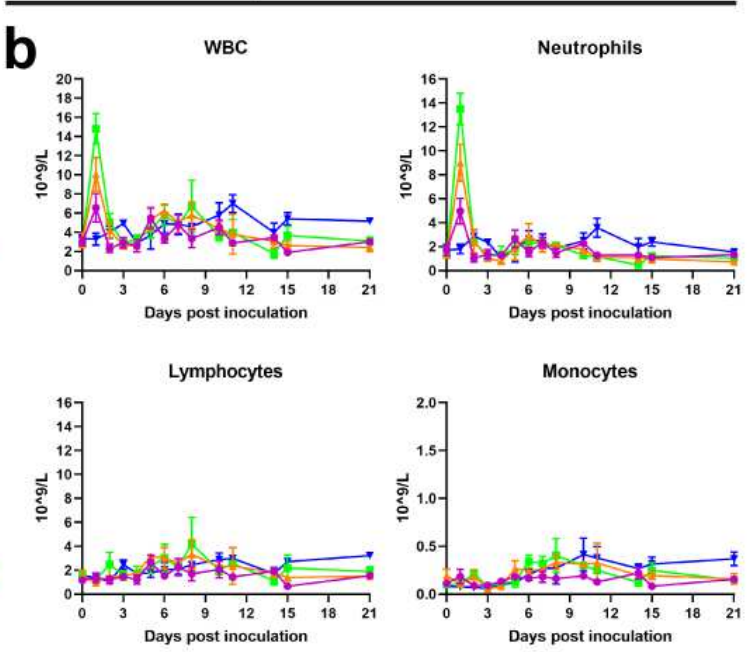

d
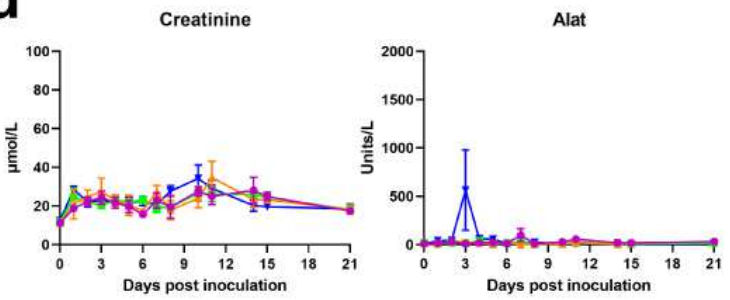

Alkaline Phosphatase

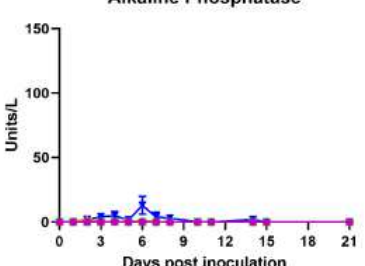

Urea

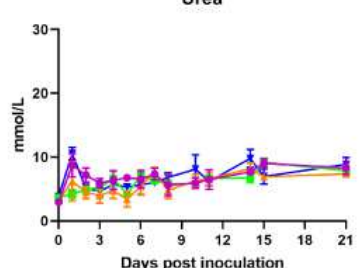

- WT RVFV-74HB59 (10^7) - hRVFV-4s $(10 \wedge 6)$

- hRVFV-4s $(10 \wedge 7)$

Figure 4. Short term neutrophilia following hRVFV-4s inoculation and no changes in blood chemistry following either

vRVFV-4s or hRVFV-4s inoculation. WBC, neutrophil, lymphocyte and monocyte counts measured in blood collected from animals from experiment 1 (a) and animals from experiment 2 (b) at the indicated time points. Creatinine, ALAT, alkaline phosphatase (ALP) and urea levels in plasma measured in animals from experiment 1 (c) and animals from experiment 2 (d), at the indicated time points. In the first week post inoculation, blood samples were obtained every other day of an individual animal (Fig 1). Averages and SEM are presented for each group. No statistical analysis could be performed due to the low number of 


\section{Absence of viremia in RVFV-4s inoculated animals}

169 One of the key safety parameters for live-attenuated vaccines is absent or limited vaccine virus viremia in the 170 vaccinated individual, thereby reducing or even preventing unwanted introduction of the vaccine in the environment, 171 either through shedding or via vectors, such as mosquitoes. To investigate if RVFV-4s induces viremia in marmosets, plasma samples were collected at regular timepoints after inoculation and tested for the presence of viral RNA and infectious virus. In both experiments, high levels of viral RNA and infectious virus were detected in plasma of marmosets inoculated with the parent viruses at 2-4 days post inoculation. Both viral RNA and infectious virus gradually decreased to undetectable levels at 11 and $7 \mathrm{DPI}$, respectively (Fig 5a-d). Peak viremia in animals inoculated with wild-type virus coincided with increases in abdominal body temperatures (Fig. 3). In the vRVFV-4s

177 and hRVFV-4s inoculated animals viral RNA was detected at DPI 1 and gradually declined to a level below the 178 detection limit around DPI 5. The total genome copy numbers detected at this time point $\left(<10^{\wedge} 7 / \mathrm{ml}\right)$ were 179 approximately three logs lower than the total number of genome copies present in the inoculum (>10^10/ml). No 180 increases in viral RNA levels were detected in these animals, suggesting that RVFV-4s inoculation does not result 181 in vaccine virus viremia. This is supported by the lack of detectable infectious vaccine virus in blood collected from RVFV-4s inoculated animals (Fig 5c and d). 


\section{Experiment 1}

a

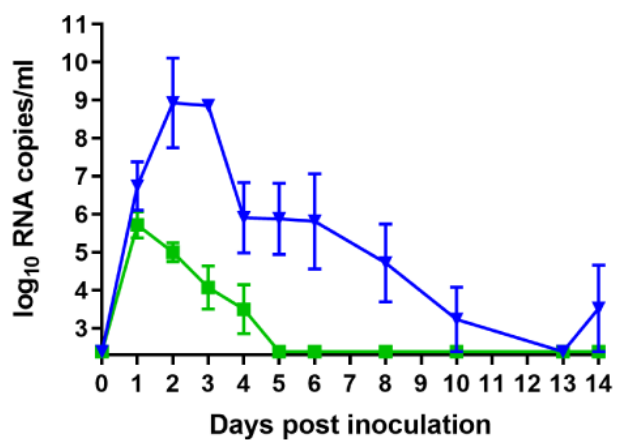

C

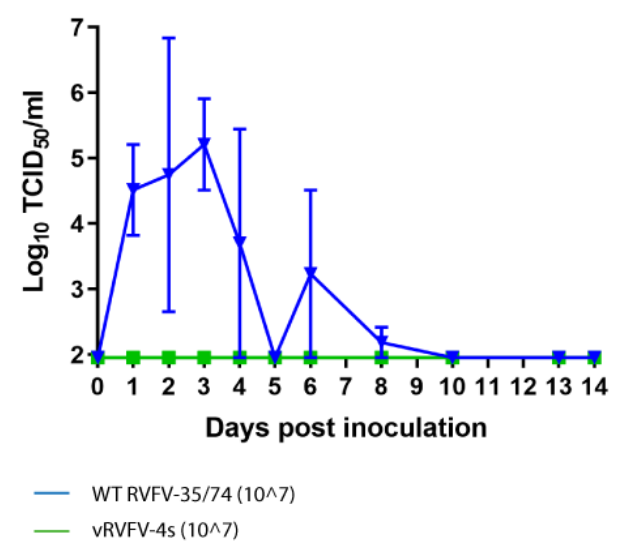

Experiment 2

b Viral RNA Plasma

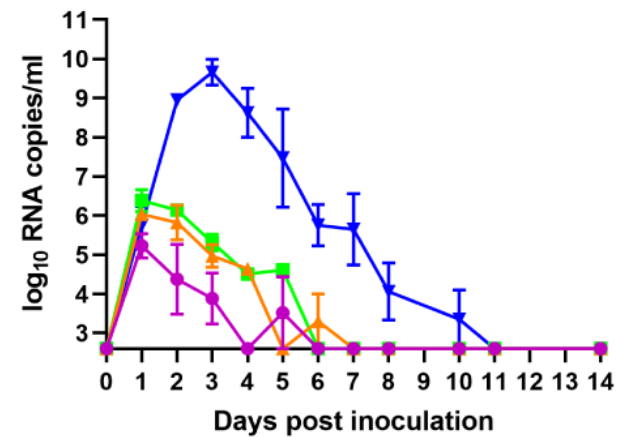

d Infectious virus plasma

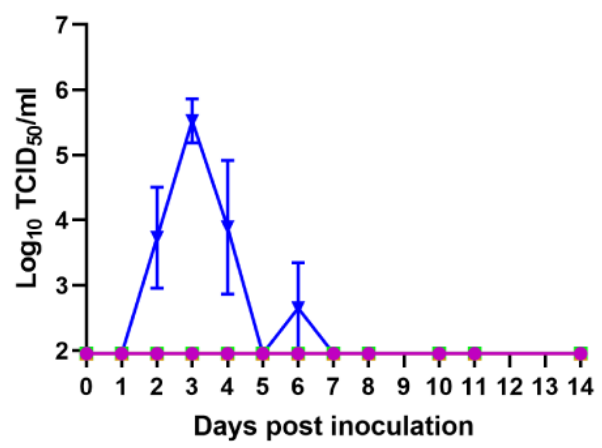

$\begin{array}{ll}-\quad \text { WT RVFV-74HB59 (10^7) } & - \text { hRVFV-4s }\left(10^{\wedge} 6\right) \\ -\operatorname{hRVFV}-4 \mathrm{~s}\left(10^{\wedge} 7\right) & - \text { hRVFV-4s }\left(10^{\wedge} 5\right)\end{array}$

Figure 5. No infectious virus recovered from plasma of RVFV-4s inoculated marmosets. Plasma samples were assessed for the presence of viral RNA by RT-qPCR $(\mathbf{a}, \mathbf{b})$ and for infectious virus by virus isolation (c, $\mathbf{d})$. Samples that tested negative are depicted at the detection limit of the PCR $(2.6 \log 10 \mathrm{RNA}$ copies $/ \mathrm{ml})$ or virus isolation $\left(1.95 \log 10 \mathrm{TCID} \mathrm{D}_{50} / \mathrm{ml}\right)$. In the first week post inoculation, the animals were allotted to one of two sampling groups. Animals in each group were bled every other day to minimize withdrawal volumes (see Fig 1).

\section{Absence of RVFV-4s dissemination to RVFV target organs}

To determine if RVFV-4s vaccine viruses disseminated in the inoculated marmosets, we assessed a broad range of organs for the presence of viral RNA. Of note, most of the organs were obtained at necropsy at 2, 3 or 4 weeks post inoculation, at which time viremia of the parent viruses had subsided. Nevertheless, in animals inoculated with the parent viruses (experiments $1 \& 2$ ) viral RNA was detected in liver, brain, spleen, kidney, lymph node, heart and adrenal gland samples (Figs. 6a and b). Furthermore, a few bone marrow, retina and ovary samples were positive for viral RNA, albeit at a very low level. Notably, from the animals that were euthanized upon reaching a humane 
end-point, a very high PCR signal was obtained from a kidney sample of animal M12030 (experiment 1) and a moderate signal for brain samples of animals M16017 and M16040 (experiment 2). Histopathological and immunohistochemical examination revealed an extensive tubulo-interstitial nephritis in animal M12030 with strong RVFV immunolabelling of the renal tubular epithelium and proteinaceous/ cellular casts within the tubuli. (Supplementary Fig.1, panel A and B). Animals M16017 an M16040 showed a multifocal meningo-encephalitis with immunostaining of neurons and neuronal processes throughout the brain (Supplementary Fig 1, panel C and D). In the vRVFV-4s inoculated animals no viral RNA was detected in liver, spleen, kidney, heart and adrenal gland samples, but viral RNA was detected in various lymph node samples and occasionally in spleen samples. Remarkably, the overall number of RVFV-4s RNA positive lymph node samples was higher in experiment 1 compared to experiment 2 .

210

\section{Absence of hRVFV-4s dissemination to the environment}

212 To investigate vaccine virus dissemination to the environment, rectal and oral swabs were obtained at various time point from all animals that were part of experiment 2 (Fig. 1). No swab samples were collected during experiment 1. PCR analysis revealed low levels of viral RNA in both oral and rectal swab samples collected from animals inoculated with the wild-type viruses, whereas no viral RNA was detected in any sample collected from hRVFV-4s inoculated animals (Fig. $6 \mathrm{c}$ and d). These results suggest that hRVFV-4s does not disseminate to the environment. 
a

PCR organs (Exp. 1)

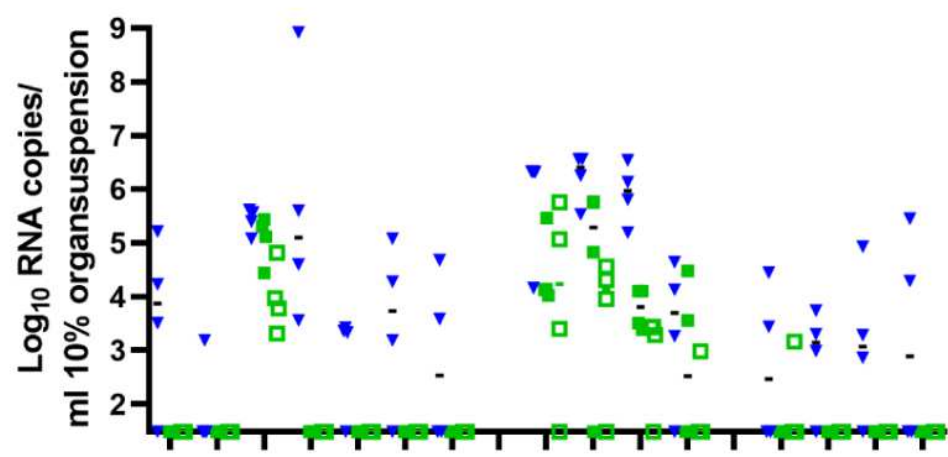

3.

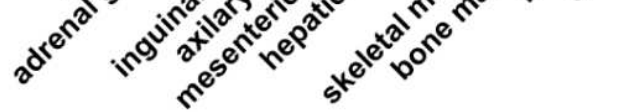

VRVFV-35/74 (2 weeks)

- vRVFV-4s (2 weeks)

ㅁ vRVFV-4s (4 weeks)

b

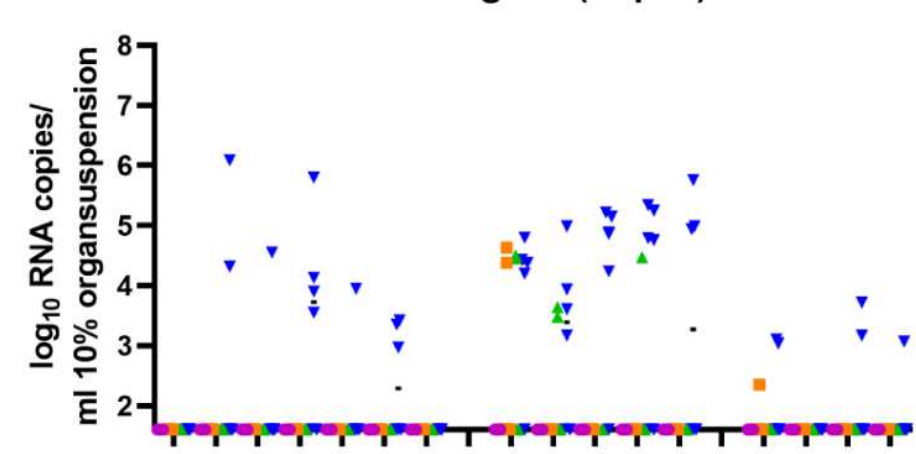
at the limit of detection.

Induction of a robust immune response
C

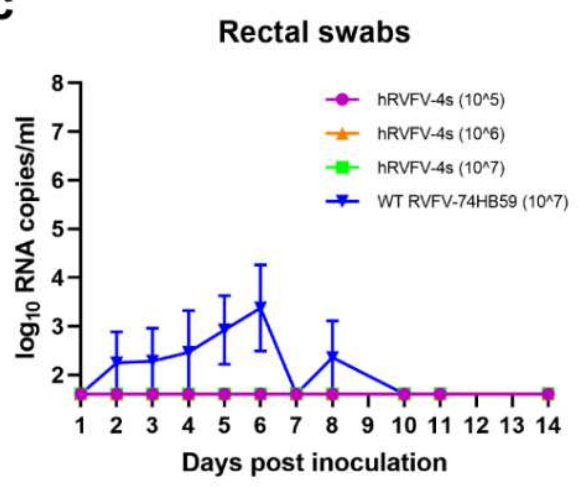

d

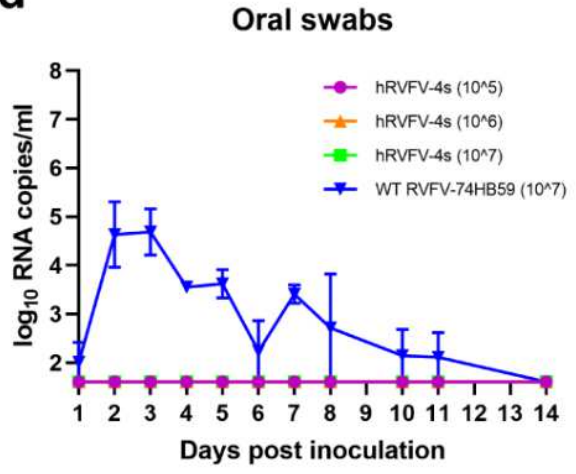

Figure 6. RVFV-4s does not disseminate to RVFV target organs, while the virus is most likely carried by phagocytes to lymphoid organs. Organ suspensions of marmosets from experiment 1 (a) and experiment 2 (b) as well as rectal (c) and oral swabs (d) of animals from experiment 2 were assessed for the presence of viral RNA by RT-qPCR. The x-axes cross the y-axes 
As neutralizing antibodies are the only known correlate of protection for RVFV, we assessed those responses in both experiments. Starting from DPI 7, neutralizing antibodies were detected in both vRVFV-4s and hRVFV-4s inoculated animals, and neutralizing antibody levels increased up to 3 weeks post inoculation (Fig. 7). In experiment 2, in which several RVFV-4s doses were assessed, lower levels of neutralizing antibodies were observed in animals inoculated with $10^{\wedge} 5 \mathrm{TCID}_{50}$, relative to the higher dose groups. No differences were observed between animals vaccinated with $10^{\wedge} 6$ and $10^{\wedge} 7 \mathrm{TCID}_{50}$. As expected, all parent virus-inoculated animals developed high levels of neutralizing antibodies. Remarkably, the overall levels of neutralizing antibodies of the parent virus-inoculated animals were only slightly higher compared to those detected in the vRVFV-4s and hRVFV-4s high-dose groups.

a

VNT Exp. 1

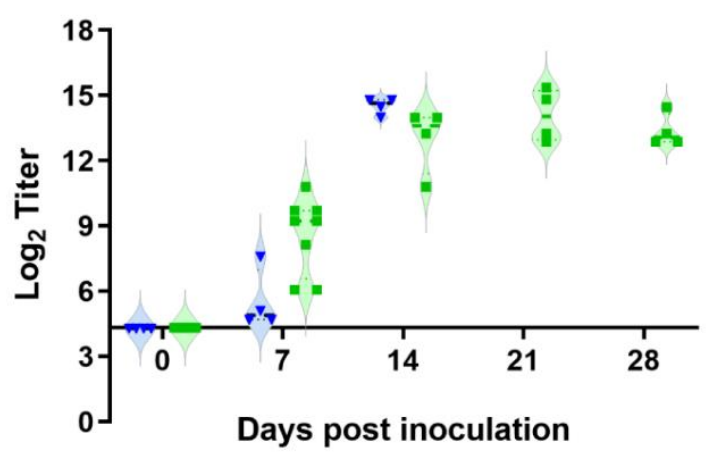

WT RVFV-35/74 (10^7) VRVFV-4s (10^7) b

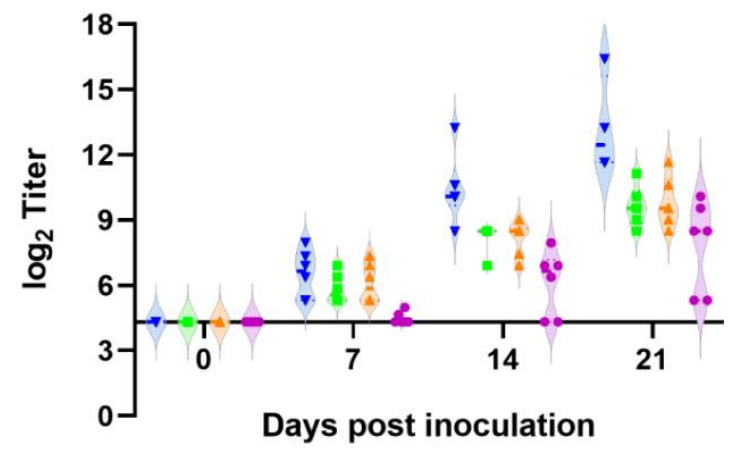

Days post inoculation

Figure 7. RVFV-4s inoculation results in the induction of a robust neutralizing antibody response in marmosets. Plasma samples of experiment 1 (a) and experiment 2 (b) were assessed for the presence of RVFV-specific neutralization antibodies by VNT assay. Violin plots are presented with $x$-axes crossing the $y$-axes at the limit of detection. 


\section{Discussion}

In earlier work, we have demonstrated that the vRVFV-4s vaccine candidate does not disseminate in inoculated ruminants and is not shed from these animals to the environment, while inducing protective immune responses after a single vaccination ${ }^{16}$. Anticipating on accidental exposure of humans to this veterinary vaccine, we evaluated its safety in the most susceptible NHP model, the common marmoset. In a similar animal model, we evaluated the safety of the hRVFV-4s candidate human vaccine, in preparation of a first-in-human clinical trial.

For live-attenuated vaccines it is of utmost importance to demonstrate the absence of virulence and inability to spread to the environment. The absence of viremia in RVFV-4s inoculated marmosets highlights that the risk of spread of the vaccine virus to mosquito vectors and subsequent spread to naive hosts is negligible. This is in agreement with our previous observation of absence of viremia in ruminants ${ }^{16}$. Furthermore, no virus was detected in the swab samples, as part of the sample panel of experiment 2 (hRVFV-4s). Of note, the levels of viral RNA in swabs were low for the wild-type virus, in line with the inability of RVFV to spread directly from animal to animal without involvement of mosquito vectors ${ }^{26}$.

While no infectious vaccine virus could be recovered from inoculated marmosets, viral RNA was detectable during the first days after inoculation in plasma samples and in lymphoid organs at the time of necropsy. It is important to realize that about $10^{\wedge} 10$ genome copies were administrated per individual animal $\left(1\right.$ TCID $_{50}$ was previously shown to be equivalent to about 1000 genome copies ${ }^{16}$ ). Taking into account the small size of a marmoset, $350-400$ grams with about $35 \mathrm{ml}$ total blood volume, it is not surprising to detect viral RNA in the circulation early after inoculation. The decline of viral RNA levels in plasma samples at 2 DPI contrasts the exponentially increased RNA levels detected in marmosets inoculated with wild-type virus at 2 DPI. These observations suggest limited, or even absent replication of RVFV-4s in vivo. The presence of vaccine virus RNA in lymphoid organs at the time of necropsy can be explained by phagocytosis of vaccine virus, genomic RNA and/or phagocytosis of infected cells by migrating cells (macrophages, dendritic cells, neutrophils). Notably, the number of lymphoid organs positive for viral RNA in experiment 1 was higher compared to experiment 2 . The different inoculation routes (combined IM/SC versus IM) or the different genetic lineages of the vaccine strains used in the independent experiments may be a factor explaining this finding ${ }^{27}$.

Whereas no untoward effects or inoculation site reactions were noted in RVFV-4s inoculated animals, a dose-dependent body temperature increase was observed consistently in these animals at 24 hrs post inoculation. Importantly, in both experiments, the expected temperature increase in animals inoculated with wildtype virus followed a different pattern and became apparent at 2-3 DPI, which correlated with peak viremia. Most likely, the vaccine viruses were efficiently detected by the marmoset's innate immune system and triggered innate immune 
responses with associated pyrexia. It is unlikely that other components besides the virus, like media- or cell components, induced these pyrogenic reactions, since the inocula containing the wild-type virus was prepared using the same cells and culture medium. Considering that the NSs protein, which is only present in the parent viruses, is known to efficiently counteract early innate immune responses ${ }^{8}$, we hypothesize that NSs delays the induction of an innate immune response in marmosets inoculated with wild-type RVFV. It is plausible that also other liveattenuated RVFV candidate vaccines lacking NSs induce a short-lived pyrexia, however, such responses may not have been noted in earlier studies as body temperatures were not measured continuously ${ }^{28}$, as was done in the present study. Overall, the dose-dependent pyrexia could be considered a mild side effect of the vaccines but also suggests that the vaccines induce potent innate immune responses, likely supporting subsequent adaptive responses.

As part of the safety analysis, we also assessed blood cell counts in both experiments. In general, the overall results with respect to blood cell counts of experiment 1 and experiment 2 were similar, although we observed one striking difference. In experiment 1, increases in total WBC and neutrophil levels were observed in wild-type 35/74 inoculated animals whereas no such changes were observed in vRVFV-4s inoculated animals, while in experiment 2, no increases in WBC and neutrophil levels were observed in marmosets inoculated with the wild-type virus, whereas dose-dependent increases were observed in hRVFV-4s inoculated animals. Remarkably, increased neutrophil levels were detected on only one day. We do not have a clear explanation for the differences in neutrophil counts between the experiments, but it might be related to the differences in genetic background of the vaccines and wild-type viruses ${ }^{27}$. Nevertheless, the data underscore that the hRVFV-4s vaccine is recognized efficiently by the marmoset's innate immune system. Whether memory cellular immune responses also differ after inoculation with the different vaccines awaits further study.

Altogether, the present work emphasises that both vRVFV-4s and hRVFV-4s have a high safety profile in the marmoset model and that both vaccine viruses induce significant immune responses following vaccination, supporting further development of both vaccines for animal and human vaccination. 


\section{Materials and methods}

296

297

298

299

300

301

302

303

304

305

306

307

308

309

310

311

312

313

314

315

316

317

318

319

320

321

322

323

324

325

\section{Ethical approval}

The study was reviewed and approved by the Dutch "Centrale Commissie Dierproeven" (AVD5020020174224) according to Dutch law, article 10a of the "Wet op de Dierproeven". The specific experiments were approved by BPRC's Animal Welfare Body (IvD).

\section{Animals and housing}

The experiments were carried out with adult common marmosets (Callithrix jacchus) of $>350$ gram. In experiment 1, 12 female animals were enrolled whereas in experiment 2, 16 male and 8 female animals were used. Animals were identified by a coloured label on a chain and an implanted microchip. The animals underwent a full physical examination, including faecal screening for pathogens and clinical chemistry and haematology evaluation before enrolment into the study. Only healthy individuals with normal clinical chemistry and haematology values were selected. Animals were housed in pairs in hBSL-3 facilities during the study period. The animals were offered a daily diet which was optimized for marmosets at the BPRC. Enrichment was offered daily, and drinking water was available ad libitum.

\section{Cells and Viruses}

Culture media and supplements were obtained from Gibco unless indicated otherwise. BHK-21 and BSR-T7 cells were maintained in Glasgow minimum essential medium (GMEM) supplemented with 4\% tryptose phosphate broth, $1 \%$ minimum essential medium nonessential amino acids (MEM NEAA), 1\% antibiotic/antimycotic (a/a) and 5\% foetal bovine serum (FBS), at $37^{\circ} \mathrm{C}$ with $5 \% \mathrm{CO}_{2}$. VERO WHO cells were maintained in VP-SFM supplemented with $2 \%$ L-glutamine and $0.01 \%$ Gentamycin (Sigma). A master seed virus (MSV) batch of vRVFV-4s (previously referred as RVFV-LMMS delNSs $^{13,16}$ was prepared by infecting BSR-T7 cells with plaque-purified seed virus, cultured in complete medium (GMEM supplemented with 5\% FCS [SAFC], 4\% TPB, 0.01\% Gentamycin and 1\% NeAA) at a multiplicity of infection (MOI) of 0.002 as described previously ${ }^{16}$. A pre-seed batch of hRVFV-4s was used to amplify the virus in Vero WHO cells. Wild-type recRVFV-35/74 and wild-type recRVFV-74HB59 were produced in BHK-21 cells, respectively BSR-T7 cells ${ }^{29}$.

\section{Inoculation}

Animals of experiment 1 received a combined subcutaneously (SC) (between shoulder blades) and IM (upper left leg) inoculation of $500 \mu \mathrm{l}$ at each site with a total of $10^{\wedge} 7 \mathrm{TCID}_{50} \mathrm{VRVFV}-4 \mathrm{~s}$ or parent virus. In experiment 2 , all 
animals received two IM (upper legs) inoculations of $500 \mu \mathrm{l}$ at each site (left and right leg) with a total of $10^{\wedge} 5,10^{\wedge} 6$ or $10^{\wedge} 7$ TCID50 of hRVFV-4s or $10^{\wedge} 7 \mathrm{TCID}_{50}$ of parent virus RVFV-74HB59.

\section{Blood sampling}

Blood samples were collected daily in the first week after inoculation. To avoid exceeding the monthly maximal amount of blood ( $1 \%$ of body weight) to be drawn, half of the animals were bled on days 1,3 and 5 , the other half on days 2, 4 and 6. Additional blood samples were taken as presented in Fig. 1.

\section{General behaviour and clinical signs}

The animals were monitored for clinical signs twice per day. Animal caretakers were blinded for which animal received the high, medium or low dose of the vaccine. Because animals that received the wild-type parent viruses had to be kept separate from animals that received the vaccines, the animal caretakers were not blinded for the wild-type groups. Abnormalities were communicated immediately to the principle investigator. When an animal showed signs of disease during the day, an extra observation was scheduled in the evening. The following clinical parameters were monitored: Appetite, stool consistency, general behaviour, fever (measured via a telemetric device), anorexia, dehydration, vomiting, drooling, ataxia, horizontal nystagmus, head-pressing, tremors, shallow breathing, blood in urine, foaming at mouth, seizures, activity/lethargy, hunched posture in combination with ruffled/unkept fur (see appendix 1). These clinical signs were described previously in NHPs which succumbed after exposure to RVFV25.

\section{Body weights and temperatures}

Animals were captured in a perspex cylinder and weighed as such. A telemetric device (AnipillV2 telemetry system (BodyCAP, Hérouville Saint Clair, France) was surgically placed in the abdominal cavity at 4 weeks before inoculation. This device measures the body temperature every 15 mins and transfers data to a receiver enabling monitoring of fever development in real-time.

\section{Isolation and cryopreservation of serum and EDTA plasma}

To collect blood samples, animals were sedated with Alfaxan (12 mg/kg, IM route). Blood was drawn from the femoral vein in the groin using aseptic techniques using a Vacutainer blood collection system (Becton Dickinson, Vacutainer systems). EDTA tubes with collected blood were centrifuged for $10 \mathrm{~min}$ at 1,000 $\mathrm{g}$ followed by collection of plasma. A volume of $125 \mu$ l plasma was used for clinical chemistry measurements. The remaining material was 
stored at $-80^{\circ} \mathrm{C}$ for virus detection via PCR and for the detection of RVFV antibodies. Upon euthanasia, $2 \mathrm{ml}$ EDTA blood and plain blood samples were collected. Serum samples were prepared by centrifugation of clotted blood for $10 \min (1,000-1,300 \times g)$, serum was stored at $-20^{\circ} \mathrm{C}$.

\section{Clinical chemistry and hematology}

At indicated time points, haematology and clinical chemistry levels were measured. Hematology parameters were measured in replenished EDTA blood with a Sysmex XT-2000iV Automated Hematology Analyzer (Sysmex® Corporation of America). Clinical chemistry parameters were measured in $125 \mu$ EDTA plasma samples with a Cobas Integra 400 plus machine (Roche Diagnostics).

\section{Euthanasia and necropsy}

At the end of the study (day 14/15 or 28 in experiment 1, and day 21 in experiment 2) or upon reaching a humane end-point, animals were euthanized by intramuscular injection of an anesthetic dose of Alfaxan/Ketamin (10 mg/kg, IM route) followed by an overdose of barbiturate (Pentobarbital, 70-100 mg/kg) injected into a vein in the groin. Complete necropsy was performed on all euthanized animals. Tissues were examined for gross pathology, frozen in liquid nitrogen or fixed in $4 \%$ buffered formaldehyde for maximally $48 \mathrm{~h}$. Formalin fixed tissues were processed further by dehydration and embedding into paraffin.

\section{Oral and anal swabs}

Oral and anal swabs were collected using FLOQSwabs® 516CS01 Ultra Minitip Flocked Swab with 100mm Breakpoint (FLOQSwabs® COPAN Diagnostics Inc). Oral swabs were inserted into the oral cavity and the inside of each cheek was swabbed for a few seconds. The tip of each swab was placed into a $14 \mathrm{ml}$ tube containing $2 \mathrm{ml}$

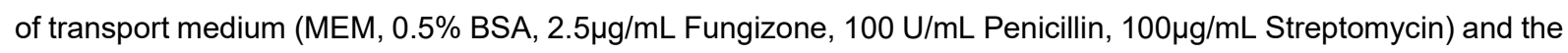
applicator stick was broken off. Vials with medium and swabs were vortexed for $20 \mathrm{~s}$. Supernatants were centrifuged for $5 \mathrm{~min}(750 \times \mathrm{g})$ and stored in at least 2 aliquots at $-80^{\circ} \mathrm{C}$. Anal swabs were placed in transport medium and handled as described above. Possible debris was pelleted by centrifugation (750 x g, 5 min) and supernatants collected and stored in at least 2 aliquots at $-80^{\circ} \mathrm{C}$.

\section{Detection of viral RNA in plasma and organ suspensions}

Frozen organ samples were thawed and subsequently homogenised using IKA Ultra Turrax Tube DT-20 in the presence of $\mathrm{CO}_{2}$-Independent Medium (CIM) supplemented with 1\% a/a to generate $10 \%$ organ suspensions. The 
suspensions were transferred to $15 \mathrm{ml}$ Falcon tubes and cell debris was removed by centrifugation for $15 \mathrm{~min}$ at $4952 \times \mathrm{g}$. Organ suspensions $(200 \mu \mathrm{l})$ or plasma samples $(25 \mu \mathrm{l})$ obtained in Experiment 1 were added to $50 \mu \mathrm{l}$ Proteinase K (5 $\mu \mathrm{g} / \mathrm{ml}$, Sigma). Next, $200 \mu \mathrm{l}$ AL buffer (Qiagen), supplemented with $2 \mu$ l polyadenylic acid A (5 $\mathrm{mg} / \mathrm{ml}$, Sigma) was added, after which the samples were thoroughly mixed and incubated at $56^{\circ} \mathrm{C}$ for $15 \mathrm{~min}$. Subsequently, $250 \mu \mathrm{l} 99 \%$ ethanol was added and RNA was isolated using the Qiagen RNeasy kit according to the manufacturer's protocol. Organ suspensions $(500 \mu \mathrm{l})$ or plasma samples $(25 \mu \mathrm{l})$ obtained in Experiment 2 were added to $2.5 \mathrm{ml}$ NucliSENS easyMAG Lysis Buffer (Biomérieux, Marcy-l'Étoile, France), after which RNA was extracted using the NucliSENS easyMAG (Biomérieux) according to manufacturer's protocol. In both experiments five $\mu$ of the RNA was used in a RT-qPCR using the The LightCycler RNA Amplification Kit HybProbe (Roche, Almere, the Netherlands). Primers and probes were purchased from IDT. Forward primer: 5'AAAGGAACAATGGACTCTGGTCA-3', reverse primer: 5'-CACTTCTTACTACCATGTCCTCCAAT-3'; Probe: 5'6FAM-AAAGCTTTGATATCTCTCAGTGCCCCAA-TMR-3'. Cycling conditions were as follows: $45^{\circ} \mathrm{C}$ for 30 min, $95^{\circ} \mathrm{C}$ for $5 \mathrm{~min}, 45$ cycles of $5 \mathrm{~s}$ at $95^{\circ} \mathrm{C}$ and $35 \mathrm{~s}$ at $57^{\circ} \mathrm{C}$, followed by cooling down to $30^{\circ} \mathrm{C}$.

\section{Virus isolation}

Virus isolations were performed on PCR-positive plasma samples with a threshold above $10^{\wedge} 5$ RNA copies/ml as this has been previously shown to be a cut-off point below which no live virus can be detected ${ }^{19}$. Virus isolations were performed by serial dilution in complete $\mathrm{CO}_{2}$-indepentent medium (CIM; supplemented with $5 \%$ FBS and $1 \%$ a/a) supplemented with $3.5 \mathrm{IU} / \mathrm{ml}$ heparin. Subsequently, the virus dilutions were incubated with BHK-21 cells. After $1.5 \mathrm{~h}$ incubation at RT, the inocula were replaced by fresh medium and after 5 days of culturing the cells at $37^{\circ} \mathrm{C}$ and $5 \% \mathrm{CO}_{2}$ cytopathic effects were scored.

\section{Virus neutralisation test and ELISA}

Serum RVFV neutralising antibodies were measured using a virus neutralization test (VNT) ${ }^{30}$. Briefly, serial dilutions $(50 \mu \mathrm{l})$ of heat-inactivated sera $\left(2 \mathrm{~h}, 56^{\circ} \mathrm{C}\right)$ were incubated with $50 \mu \mathrm{l}$ of RVFV-4SeGFP $\left(10^{3.6} \mathrm{TCID} 50 / \mathrm{ml}\right)$ for $2 \mathrm{~h}$ at $\mathrm{RT}$. Subsequently, 20,000 BHK-21 cells (in $50 \mu \mathrm{l}$ ) were added to each well. Plates were incubated for 2 days at $37^{\circ} \mathrm{C}$ and $5 \% \mathrm{CO}_{2}$ and scored using an EVOS-FL microscope (Life Technologies). $\mathrm{VNT}_{50}$ titres were calculated using the Spearman-Kärber algorithm. Presence of RVFV nucleoprotein-specific antibodies in sera was determined using the ID Screen® Rift Valley Fever Competition ELISA (ID-Vet, Montpellier, France). 


\section{Histology and immunohistochemistry}

Paraffin embedded tissues were cut into $4 \mu \mathrm{m}$ sections, collected on silane-coated glass slides and dried for at least $48 \mathrm{~h}$ in a $37^{\circ} \mathrm{C}$ incubator. After deparaffinization and rehydration in graded alcohols, sections were stained routinely with haematoxylin and eosin (H\&E) or immunostained for RVFV antigen. For immunostaining, epitopes were retrieved by autoclaving for 5 minutes at $121^{\circ} \mathrm{C}$ in $\mathrm{pH} 6$ citrate buffer (Antigen unmasking solution, Vector Laboratories). RVFV Gn-specific monoclonal antibody 4-D4 was used as primary antibody. Specificity of the immunostaining was confirmed with 2 other mAbs directed against the Gc and N protein of RVFV. HRP-conjugated anti-mouse IgG polymer (Invitrogen) was used as secondary antibody and DAB+ as substrate (Dako, Agilent) Sections were counterstained with haematoxylin.

\section{Data availability}

All data necessary to interpret, replicate and build upon the methods or findings reported in the article are provided in this article.

\section{Acknowledgements}

We thank the animal caretakers for providing assistance in the animal trials. We thank Dr. Schmaljohn (USAMRIID, Fort Detrick, MD) for providing the 4-D4 mAb and Prof. Klaus Conzelmann (Ludwich-Maximilians-Universität, München) for providing the BSR-T7 cells. Experiment 1 was performed within the scope of the Castellum program, funded by the former Ministry of Economic Affairs. Experiment 2 was performed within the LARISSA project and was funded by the Coalition for Epidemic Preparedness Innovations (CEPI).

\section{Competing interests}

P.J.W.S, and J.Ko. are inventors of WIPO Patent Application WO/2014/189372 "Bunyaviruses with segmented glycoprotein genes and methods for generating these viruses", owned by BunyaVax B.V. CP is chief executive officer and P.J.W.S. is Chief Scientific Officer/Chief Operations Officer of BunyaVax. The remaining authors declare no competing interests.

\section{Author contributions}

P.J.W.S., S.W., and J.Ko. conceived, designed and produced the vaccine. P.J.W.S., J.Ko., L.K., P.M, G.K., B.V, W.B., and E.V. designed the animal experiments. P.J.W.S., P.M., G.K., B.V., Z.F., D.M., N.D., J.Ka., S.W., W.B. and L.K. performed infections, analytical assays, necropsies and histopathology. P.J.W.S., P.M., G.K., B.V., W.B., 
the manuscript.

Moutailler, S., Krida, G., Schaffner, F., Vazeille, M. \& Failloux, A. B. Potential vectors of Rift Valley fever virus in the Mediterranean region. Vector borne and zoonotic diseases 8, 749-753, doi:10.1089/vbz.2008.0009 (2008).

2 Turell, M. J. et al. Vector competence of selected African mosquito (Diptera: Culicidae) species for Rift Valley fever virus. Journal of medical entomology 45, 102-108, doi:10.1603/00222585(2008)45[102:vcosam]2.0.co;2 (2008).

3 Bron, G. M. et al. Over 100 Years of Rift Valley Fever: A Patchwork of Data on Pathogen Spread and Spillover. Pathogens 10, doi:10.3390/pathogens10060708 (2021).

4 Ikegami, T. \& Makino, S. The pathogenesis of Rift Valley fever. Viruses 3, 493-519, doi:10.3390/v3050493 (2011)

5 Baudin, M. et al. Association of Rift Valley fever virus infection with miscarriage in Sudanese women: a cross-sectional study. Lancet Glob Health 4, e864-e871, doi:10.1016/S2214-109X(16)30176-0 (2016).

6 McMillen, C. M. et al. Rift Valley fever virus induces fetal demise in Sprague-Dawley rats through direct placental infection. Sci Adv 4, eaau9812, doi:10.1126/sciadv.aau9812 (2018).

7 Oymans, J., Wichgers Schreur, P. J., van Keulen, L., Kant, J. \& Kortekaas, J. Rift Valley fever virus targets the maternal-foetal interface in ovine and human placentas. PLoS Negl Trop Dis 14, e0007898, doi:10.1371/journal.pntd.0007898 (2020).

8 Ly, H. J. \& Ikegami, T. Rift Valley fever virus NSs protein functions and the similarity to other bunyavirus NSs proteins. Virology journal 13, 118, doi:10.1186/s12985-016-0573-8 (2016).

9 Won, S., Ikegami, T., Peters, C. J. \& Makino, S. NSm protein of Rift Valley fever virus suppresses virusinduced apoptosis. J Virol 81, 13335-13345, doi:10.1128/JVI.01238-07 (2007).

10 Kading, R. C. et al. Deletion of the NSm virulence gene of Rift Valley fever virus inhibits virus replication in and dissemination from the midgut of Aedes aegypti mosquitoes. PLoS Negl Trop Dis 8, e2670, doi:10.1371/journal.pntd.0002670 (2014).

11 Weingartl, H. M. et al. Rift Valley Fever Virus Incorporates the 78 kDa Glycoprotein into Virions Matured in Mosquito C6/36 Cells. PloS one 9, e87385, doi:10.1371/journal.pone.0087385 (2014).

12 Kreher, F. et al. The Rift Valley fever accessory proteins NSm and P78/NSm-GN are distinct determinants of virus propagation in vertebrate and invertebrate hosts. Emerging microbes \& infections 3, e71, doi:10.1038/emi.2014.71 (2014).

13 Wichgers Schreur, P. J., Oreshkova, N., Moormann, R. J. \& Kortekaas, J. Creation of Rift Valley fever viruses with four-segmented genomes reveals flexibility in bunyavirus genome packaging. Journal of virology 88, 10883-10893, doi:10.1128/JVI.00961-14 (2014).

14 Muller, R. et al. Characterization of clone 13, a naturally attenuated avirulent isolate of Rift Valley fever virus, which is altered in the small segment. Am J Trop Med Hyg 53, 405-411, doi:10.4269/ajtmh.1995.53.405 (1995).

Bouloy, M. et al. Genetic evidence for an interferon-antagonistic function of rift valley fever virus nonstructural protein NSs. Journal of virology 75, 1371-1377, doi:10.1128/JVI.75.3.1371-1377.2001 (2001).

16 Wichgers Schreur, P. J. et al. Safety and efficacy of four-segmented Rift Valley fever virus in young sheep, goats and cattle. NPJ Vaccines 5, 65, doi:10.1038/s41541-020-00212-4 (2020).

17 Wichgers Schreur, P. J. et al. A single vaccination with four-segmented rift valley fever virus prevents vertical transmission of the wild-type virus in pregnant ewes. NPJ Vaccines 6, 8, doi:10.1038/s41541020-00271-7 (2021).

18 Wichgers Schreur, P. J. et al. Safety and efficacy of four-segmented Rift Valley fever virus in young sheep, goats and cattle. NPJ Vaccines 5, 65, doi:10.1038/s41541-020-00212-4 (2020).

19 Wichgers Schreur, P. J., van Keulen, L., Kant, J. \& Kortekaas, J. Four-segmented Rift Valley fever virusbased vaccines can be applied safely in ewes during pregnancy. Vaccine 35, 3123-3128, doi:10.1016/j.vaccine.2017.04.024 (2017).

20 Wichgers Schreur, P. J., Kant, J., van Keulen, L., Moormann, R. J. \& Kortekaas, J. Four-segmented Rift Valley fever virus induces sterile immunity in sheep after a single vaccination. Vaccine 33, 1459-1464, doi:10.1016/j.vaccine.2015.01.077 (2015).

21 Estes, J. D., Wong, S. W. \& Brenchley, J. M. Nonhuman primate models of human viral infections. Nat Rev Immunol 18, 390-404, doi:10.1038/s41577-018-0005-7 (2018).

22 Morrill, J. C. et al. Pathogenesis of Rift Valley fever in rhesus monkeys: role of interferon response. Archives of virology 110, 195-212, doi:10.1007/BF01311288 (1990).

23 Peters, C. J. et al. Experimental Rift Valley fever in rhesus macaques. Archives of virology 99, 31-44, doi:10.1007/BF01311021 (1988). 
Smith, D. R. et al. Development of a novel nonhuman primate model for Rift Valley fever. Journal of virology 86, 2109-2120, doi:10.1128/JVI.06190-11 (2012).

Hartman, A. L. et al. Aerosolized rift valley fever virus causes fatal encephalitis in african green monkeys and common marmosets. Journal of virology 88, 2235-2245, doi:10.1128/JVI.02341-13 (2014). Wichgers Schreur, P. J. et al. Co-housing of Rift Valley Fever Virus Infected Lambs with Immunocompetent or Immunosuppressed Lambs Does Not Result in Virus Transmission. Frontiers in microbiology 7, 287, doi:10.3389/fmicb.2016.00287 (2016).

Ikegami, T. et al. Distinct virulence of Rift Valley fever phlebovirus strains from different genetic lineages in a mouse model. PLoS One 12, e0189250, doi:10.1371/journal.pone.0189250 (2017).

Smith, D. R. et al. Attenuation and efficacy of live-attenuated Rift Valley fever virus vaccine candidates in non-human primates. PLoS neglected tropical diseases 12, e0006474, doi:10.1371/journal.pntd.0006474 (2018).

29 Kortekaas, J. et al. Creation of a nonspreading Rift Valley fever virus. J Virol 85, 12622-12630, doi:10.1128/JVI.00841-11 (2011).

30 Wichgers Schreur, P. J., Paweska, J. T., Kant, J. \& Kortekaas, J. A novel highly sensitive, rapid and safe Rift Valley fever virus neutralization test. Journal of Virological Methods 248, 26-30, doi:https://doi.org/10.1016/j.jviromet.2017.06.001 (2017). 


\section{Supplementary Files}

This is a list of supplementary files associated with this preprint. Click to download.

- Supplementarydatav1.pdf 